\title{
Dynamical spin-electric coupling in a quantum dot
}

\section{Citation}

Levitov, L. S., and E. I. Rashba. 2003. "Dynamical Spin-Electric Coupling in a Quantum Dot." Physical Review B 67 (11) (March 24). doi:10.1103/physrevb.67.115324.

\section{Published Version}

doi:10.1103/physrevb.67.115324

\section{Permanent link}

http://nrs.harvard.edu/urn-3:HUL.InstRepos:25886935

\section{Terms of Use}

This article was downloaded from Harvard University's DASH repository, and is made available under the terms and conditions applicable to Other Posted Material, as set forth at http:// nrs.harvard.edu/urn-3:HUL.InstRepos:dash.current.terms-of-use\#LAA

\section{Share Your Story}

The Harvard community has made this article openly available.

Please share how this access benefits you. Submit a story.

\section{Accessibility}




\title{
Dynamical spin-electric coupling in a quantum dot
}

\author{
L. S. Levitov ${ }^{1}$ and E. I. Rashba ${ }^{1,2, *}$ \\ ${ }^{1}$ Department of Physics, Center for Materials Sciences \& Engineering, Massachusetts Institute of Technology, \\ Cambridge, Massachusetts 02139 \\ ${ }^{2}$ Department of Physics, SUNY at Buffalo, Buffalo, New York 14260
}

(Received 30 September 2002; published 24 March 2003)

\begin{abstract}
Due to the spin-orbital coupling in an anisotropic semiconductor quantum dot, a freely precessing electron spin produces a time-dependent charge density. This creates a sizable electric field outside the dot, leading to promising applications in spintronics. The spin-electric coupling can be employed for noninvasive single-spin detection by electrical methods. We also consider a spin relaxation mechanism due to long-range coupling to electrons in gates and elsewhere in the system, and find a contribution comparable to, and in some cases dominant over, previously discussed mechanisms.
\end{abstract}

DOI: 10.1103/PhysRevB.67.115324

PACS number(s): 72.25.Dc, 72.80.Rj, 73.21.La

Because of the spin-orbit (SO) interaction, a precessing electron spin in a semiconductor produces a time-dependent oscillating electric field along with a magnetic field. This effect is weak in single molecules, because the SO coupling is small in the inverse Dirac gap $2 m_{\mathrm{e}} c^{2} \approx 1 \mathrm{MeV}$. In semiconductors it is enhanced, since the SO splitting of the upper valence band $(0.3 \mathrm{eV}$ in $\mathrm{GaAs}, 0.9 \mathrm{eV}$ in $\mathrm{InSb})$ can reach or even exceed the energy gap size. The SO effects are further reinforced by low symmetry, ${ }^{1}$ allowing for a strong coupling of the electron spin to static and time-dependent electric fields.

We propose to employ the electric field produced by a freely precessing electron spin in a quantum dot for noninvasive single-spin detection, which may have promising applications in spintronics. ${ }^{2}$ A different idea, based on charge transport through the dot, was put forward by Engel and Loss. ${ }^{3}$ The spin-electric coupling considered below leads to novel physical effects, including a new mechanism of spin relaxation complementary to that discussed recently by Khaetskii and Nazarov. ${ }^{4}$

Low symmetry is crucial for this. While in spherically symmetric systems, such as atoms, the SO-induced orbital magnetization currents do not produce electric dipole or higher-multipole moments, in a less symmetric system the orbital currents can generate a time-varying electric field accompanying spin precession. To clarify the underlying physics and to simplify the calculations, we consider a quantum dot with highly anisotropic confinement, such as a dot created within a quantum wire.

Electron confinement in such a system is highly anisotropic. It is shallow along the wire (we call this direction $x$ ) and tight in other directions $(y$ and $z$ ). Furthermore, we assume that the wire structure in the cross section lacks inversion symmetry. The SO interaction arises in this case from two separate contributions: the confinement-enhanced bulk inversion assymmetry (BIA, Dresselhaus) and the structure inversion asymmetry (SIA, Rashba). ${ }^{5,6}$ The resulting SO interaction, linear in the electron momentum $\mathbf{k} \| \hat{\mathbf{x}}$, can be written as $\mathcal{H}_{\mathrm{SO}}=\alpha \boldsymbol{\sigma} \cdot(\mathbf{n} \times \mathbf{k})$ with the vector $\mathbf{n}$ perpendicular to the wire. We choose $\mathbf{n}=\hat{\mathbf{z}}$ which gives $\mathcal{H}_{\mathrm{SO}}=\alpha \sigma_{2} \hat{k}$ with $\hat{k}=-i \partial_{x}$. This form of SO interaction has been used for dislocations ${ }^{7}$ and quantum wires. ${ }^{8}$ The total one-dimensional (1D) Hamiltonian is

$$
\mathcal{H}_{0}=\hbar^{2} \hat{k}^{2} / 2 m^{*}+U(x)+\alpha \sigma_{2} \hat{k},
$$

with $U(x)$ the confining potential. For magnetic fields transverse to the $y$ axis, without loss of generality, we write the Zeeman interaction as $\mathcal{H}_{Z}=-\mu B \sigma_{3}$. (For the field parallel to the $y$ axis the Zeeman and SO terms commute, and thus the spin-electric coupling is absent.)

We eliminate $\mathcal{H}_{\text {SO }}$ from $\mathcal{H}_{0}$ by a canonical transformation with a unitary matrix $S=e^{i \sigma_{2} x / 2 \xi}$. Here the length $\xi$ $=\hbar^{2} / 2 m^{*} \alpha$ coincides with the characteristic size of the Datta-Das device. ${ }^{9}$ The transformation shifts $\hat{k}$ by $\sigma_{2} / 2 \xi$ and moves the SO coupling to the Zeeman term:

$$
\begin{gathered}
\mathcal{H}_{0}=\hbar^{2} \hat{k}^{2} / 2 m^{*}+U(x)-m^{*} \alpha^{2} / 2 \hbar^{2}, \\
\mathcal{H}_{Z}=-\mu B\left[\sigma_{3} \cos (x / \xi)-\sigma_{1} \sin (x / \xi)\right] .
\end{gathered}
$$

For a weak magnetic field, the Zeeman term can be treated as a perturbation. In a symmetric potential $U(x)=U(-x)$, the mean value of the second term in $\mathcal{H}_{Z}$ vanishes and the spin Hamiltonian projected onto two Kramers-conjugate states becomes diagonal:

$$
\mathcal{H}_{n}=\left\langle n\left|\mathcal{H}_{Z}\right| n\right\rangle=-\mu B\langle n|\cos (x / \xi)| n\rangle \sigma_{3},
$$

where $n$ labels the orbital wave functions $\psi_{n}(x)$. The Zeeman splitting in Eq. (3) depends on the SO coupling $\alpha$.

For narrow-gap $A_{3} B_{5}$ quantum wells, the typical values of $\alpha$ originating from SIA are about $10^{-9} \mathrm{eV} \mathrm{cm} .{ }^{10}$ However, larger values of $\alpha$ up to $3 \times 10^{-9} \mathrm{eV} \mathrm{cm}$ for $\mathrm{In}_{0.75} \mathrm{Ga}_{0.25} \mathrm{As} / \mathrm{In}_{0.75} \mathrm{Al}_{0.25} \mathrm{As}$ heterojunctions ${ }^{11}$ and 6 $\times 10^{-9} \mathrm{eV} \mathrm{cm}$ for $\mathrm{In}_{0.52} \mathrm{Al}_{0.48} \mathrm{As} / \mathrm{In}_{x} \mathrm{Ga}_{1-x} \mathrm{As}$ structures ${ }^{12}$ were reported more recently. Both experiment ${ }^{13}$ and theory ${ }^{14}$ indicate that the interface asymmetry makes an important, and maybe even dominant, contribution to $\alpha$, which can be varied by system design. With $\alpha \approx 6 \times 10^{-9} \mathrm{eV} \mathrm{cm}$ and $m * / m \approx 0.05$, we estimate the characteristic length scale as $\xi \approx 13 \mathrm{~nm}$. The dependence of $\mathcal{H}_{n}$ on the SO coupling can thus be significant for quantum dots of size comparable or 
larger than $\xi$. (In diffusive dots the SO effects are controlled by the ratio of level spacing to the SO scattering spin-flip rate. ${ }^{15}$ )

Now we consider the time-dependent electric charge density arising due to electron spin precession. It is given by the off-diagonal component in the spin element of the density matrix $N_{\downarrow \uparrow}^{(n)}(x, t)=e^{-i \omega_{Z, n} t} N_{\downarrow \uparrow}^{(n)}(x)$ with the $n$th orbital state Zeeman frequency $\omega_{Z, n}$ defined by Eq. (3). Here

$$
N_{\downarrow \uparrow}^{(n)}(x)=\left\langle\bar{\Psi}_{n \downarrow}(x) \mid \Psi_{n \uparrow}(x)\right\rangle_{\text {spin }},
$$

with $\Psi_{n \sigma}(x)$ the exact Zeeman-split Kramers-doublet spinor wave functions. The partial trace in Eq. (4) is taken over spin. To the first order in $\omega_{Z}$ one obtains

$$
N_{\downarrow \uparrow}^{(n)}(x)=2 \mu B \sum_{n^{\prime} \neq n} \frac{\psi_{n}(x) \psi_{n^{\prime}}(x)}{E_{n}-E_{n^{\prime}}}\left\langle n^{\prime}\left|\sin \left(x^{\prime} / \xi\right)\right| n\right\rangle,
$$

and the corresponding dipole moment equals

$$
P_{n}=-2 e \mu B \sum_{n^{\prime} \neq n} \frac{\left\langle n\left|x^{\prime}\right| n^{\prime}\right\rangle\left\langle n^{\prime}\left|\sin \left(x^{\prime \prime} / \xi\right)\right| n\right\rangle}{E_{n}-E_{n^{\prime}}}
$$

(here $x^{\prime}$ and $x^{\prime \prime}$ are integration variables). To complete this general discussion, let us consider free spin precession in a quantum dot holding an odd number of electrons, one electron with unpaired spin at the $n$th level, with all lower states $E_{n^{\prime}}<E_{n}$ fully filled. Taking an arbitrary superposition state for the spin configuration of the electron on the $n$th level, the time-dependent dipole is

$$
\mathbf{P}(t)=\sin \theta P_{n} \cos \left(\omega_{Z, n} t+\varphi\right) \hat{\mathbf{x}}
$$

where $\theta$ is the angle between the magnetic field and the spin precessing around it. The quantities $\omega_{Z, n}$ and $P_{n}$ are given by Eq. (3) and Eq. (6).

Now we focus on two practically interesting confinement models: a square well with hard walls, describing a quantum wire segment, and a parabolic quantum dot. For a wire segment of length $L$, using sinusoidal standing wave states, the Zeeman frequency (3) is

$$
\hbar \omega_{Z, n}(\gamma)=2 \mu B \frac{\pi^{2} n^{2} \sin \gamma}{\left(\pi^{2} n^{2}-\gamma^{2}\right) \gamma}, \quad \gamma=L / 2 \xi,
$$

with $n \geqslant 1$. (Zeros in the denominator do not cause divergence because of $\sin \gamma$.) The electric dipole (6) is

$$
P_{n}(\gamma)=-e L\left(\mu B / \Delta_{n}\right) \sum_{n^{\prime}-n=\text { odd }} \frac{\left(2^{8} / \pi^{4}\right)(2 n+1)\left(n n^{\prime}\right)^{2} \gamma \cos \gamma}{\left(n^{2}-n^{\prime 2}\right)^{3}\left\{\left[n^{2}+n^{\prime 2}-(2 \gamma / \pi)^{2}\right]^{2}-4\left(n n^{\prime}\right)^{2}\right\}},
$$

with $\Delta_{n}=E_{n+1}-E_{n}=(2 n+1) \pi^{2} \hbar^{2} / 2 m^{*} L^{2}$ the separation between the energy levels $E_{n+1}$ and $E_{n}$. The sum, evaluated exactly for $n=1$ and $\gamma \ll 1$, gives

$$
P_{1}=\frac{15-\pi^{2}}{8 \pi^{2}} \frac{\mu B}{\Delta_{1}} \frac{L}{\xi} e L .
$$

This result is similar to the matrix element of electric-dipole transitions in 3D donor centers. ${ }^{16}$ The two factors multiplying the geometric dipole $e L$ in Eq. (9) have the following meaning. The factor $\mu B / \Delta_{1} \ll 1$ reflects that the matrix element of $x$ between two Kramers-conjugate states vanishes at $B=0$ due to the time-reversal symmetry. The factor $L / \xi$ $\ll 1$ makes $P_{1}$ vanish at zero SO coupling. Despite the small factors, the electric dipole can still be much larger than the Bohr's magneton $\mu=\frac{1}{2} e \lambda_{C}$, where $\chi_{C}$ is the electron Compton length.

For a parabolic dot with a confining potential $U(x)$ $=m \omega^{2} x^{2} / 2$, the Zeeman frequency (3) is

$$
\hbar \omega_{Z, n}\left(\gamma_{2}\right)=2 \mu B e^{-\gamma_{2} / 2} L_{n}\left(\gamma_{2}\right), \quad \gamma_{2}=\frac{1}{2}\left(x_{0} / \xi\right)^{2},
$$

where $n \geqslant 0, x_{0}=(\hbar / m \omega)^{1 / 2}$, and $L_{n}$ are Laguerre polynomials. Similar to Eq. (7), the frequency (10) is a sign-changing function of the SO coupling $\alpha$, vanishing at $\alpha \rightarrow \infty$. The summation in Eq. (6), performed exactly using harmonic oscillator selection rules, gives

$$
P_{n}\left(\gamma_{2}\right)=e x_{0}(\mu B / \hbar \omega)\left(2 \gamma_{2}\right)^{1 / 2} e^{-\gamma_{2} / 2} L_{n}\left(\gamma_{2}\right) .
$$

For the ground state, the dipole moment (11) is similar to $P_{1}$ of Eq. (9) with $x_{0}$ replacing $L$.

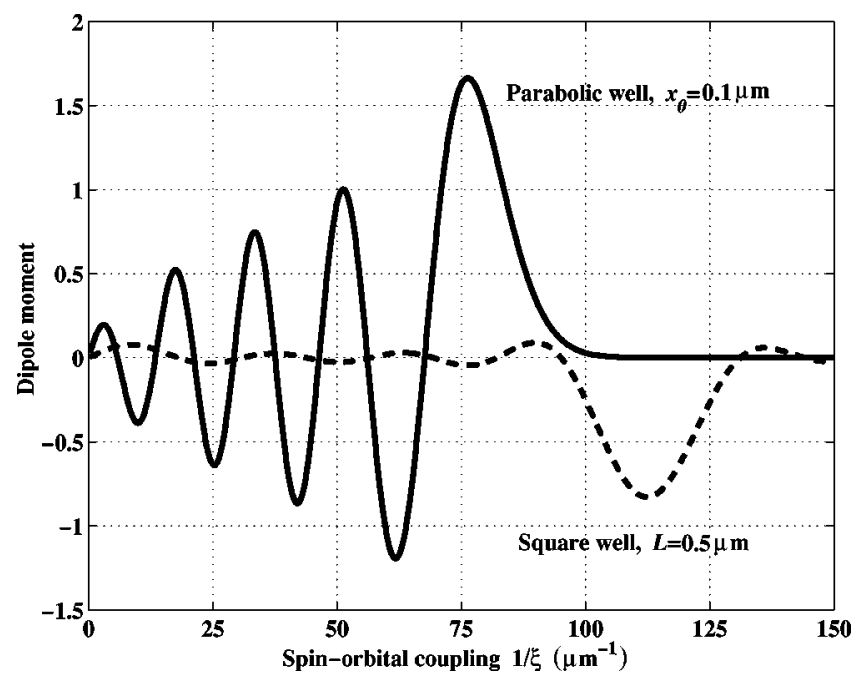

FIG. 1. Electric dipole of a Zeeman-split state vs SO coupling. The state $|n\rangle$ with $n=9$ is used. The dipole (8) for a square well is scaled by $\frac{1}{3} e L\left(\mu B / \Delta_{5}\right)$; the dipole (11) for a parabolic well is scaled by ex $(\mu B / \hbar \omega)$. 


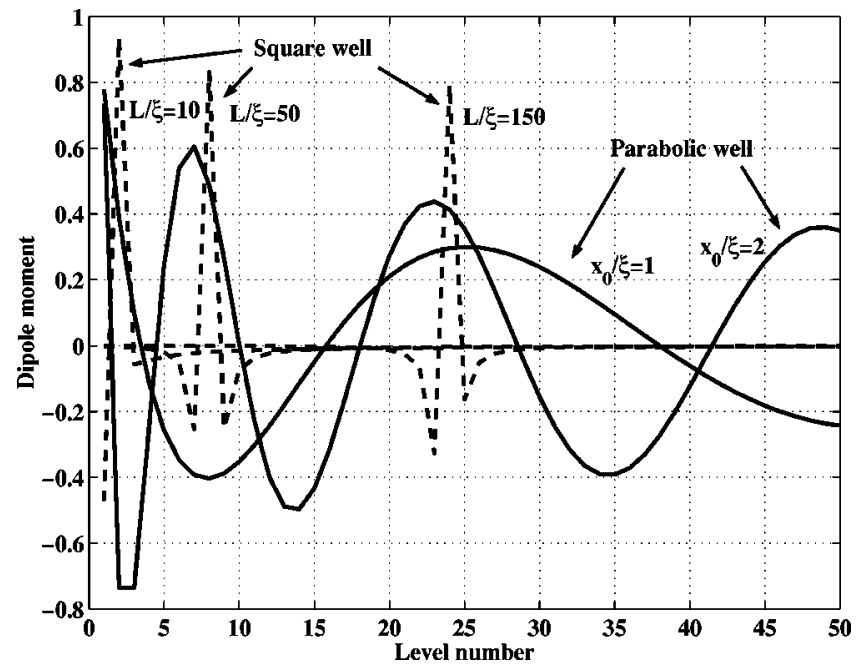

FIG. 2. Electric dipole scaled as in Fig. 1 vs level number.

The dipole moments (8) and (11) for the two models (Fig. 1) behave as follows. For a square well $P_{n}(\gamma)$ has a peak at $\gamma \approx \pi n$ of width $\delta \gamma \simeq 2 \pi$ independent of $n$. The peak is positive or negative depending on the parity of $n$, with weak oscillations on both sides. For a parabolic well the oscillations of $P_{n}(\xi)$ increase in amplitude as $\xi^{-1}$ up to $\gamma_{2} \approx 4 n$ and then abruptly disappear as $P_{n}\left(\gamma_{2}\right)$ drops.

The dependence of the dipole on the level number is quite interesting (Fig. 2). For the square well, since the dipole (8) peaks near $\gamma=\pi n, P_{n}$ is large only for specific levels, while for the harmonic potential it is a smooth oscillatory function of level number. This dramatic difference is explained as follows. In a square well each wave function is characterized by a specific wavelength, equal to $2 L / n$ for the $n$th level, while in a harmonic potential the wavelength is position dependent. The SO effect will be strong when the wavelength matches the spatial period $4 \pi \xi$ of the matrix $e^{i \sigma_{2} x / 2 \xi}$ used to gauge the SO interaction. Thus one expects the dipole in a square well to be large for states with $2 L / n=4 \pi \xi$, which is exactly the above condition $\gamma=\pi n$. In a harmonic potential, on the other hand, there should be no specific levels with enhanced dipole.

A much stronger spin-electric coupling arises for nonKramers states brought to degeneracy at the Zeeman energy $\mu B$ matching the level separation $E_{n}-E_{n^{\prime}}$. The spinelectric coupling for SO-split avoided crossings of levels with different orbital wave functions and opposite spin is different from that for a Zeeman split Kramers doublet. The matrix element of electric dipole in this case does not contain the factor $\mu B / \Delta$ that appeared in Eq. (10) due to timereversal symmetry at $B=0$. The SO-split level crossings in small elongated dots were reported by Rokhinson et al. ${ }^{17}$ and in 2D dots by Folk et al. ${ }^{18}$

For such a pair of states $\left|\psi_{n} \uparrow\right\rangle,\left|\psi_{n^{\prime}} \downarrow\right\rangle$ the energy separation in the absence of the SO coupling is $\Delta$ $=E_{n}-E_{n^{\prime}}-2 \mu B$. The avoided crossing of levels split by the SO matrix element $V$ is described by $\delta E=\left(\Delta^{2}\right.$ $\left.+4 V^{2}\right)^{1 / 2}$. The off-diagonal charge density matrix element is

$$
N_{+-}^{n n^{\prime}}(x)=u_{+} u_{-} \psi_{n}^{2}(x)+v_{+} v_{-} \psi_{n^{\prime}}^{2}(x)
$$

where

$$
u_{ \pm}=[(\delta E \pm \Delta) / 2 \delta E]^{1 / 2}
$$

and $v_{ \pm}= \pm u_{\mp}$ are the components of the two states participating in the avoided crossing. (The quantities $\psi_{n}, \psi_{n^{\prime}}$, and $V$ are real due to the absence of magnetic orbital coupling in $D=1$.) Evaluating $u_{+} u_{-}=-v_{+} v_{-}=V /\left(\Delta^{2}+4 V^{2}\right)^{1 / 2}$ we obtain

$$
N_{+-}^{n n^{\prime}}(x)=\frac{V}{\left(\Delta^{2}+4 V^{2}\right)^{1 / 2}}\left[\psi_{n}^{2}(x)-\psi_{n^{\prime}}^{2}(x)\right] .
$$

This charge density oscillates with frequency $\delta E / \hbar$.

We note that the distribution (14) possesses a dipole moment only for an asymmetric confining potential $U(x)$ $\neq U(-x)$, while the quadrupole moment $Q_{n n^{\prime}}$ $=e \int x^{2} N_{\downarrow \uparrow}^{n n^{\prime}}(x) d x$ exists even for symmetric dots:

$$
Q_{n n^{\prime}}=\frac{e V}{\left(\Delta^{2}+4 V^{2}\right)^{1 / 2}} \int x^{2}\left[\psi_{n}^{2}(x)-\psi_{n^{\prime}}^{2}(x)\right] d x .
$$

Near the resonance $\Delta \approx V$, the quadrupole moment $Q_{n n^{\prime}}$ contains no small factors and is controlled by the integrand. For a parabolic confinement potential,

$$
Q_{n n^{\prime}}=e x_{0}^{2}\left(n-n^{\prime}\right) V /\left(\Delta^{2}+4 V^{2}\right)^{1 / 2} .
$$

The factor $e x_{0}^{2} n$ has the scale of the quadrupole moment of the $n$th quantum state. The enhancement of the electrical signal from a spin precessing between two non-Kramers levels resembles a similar effect for the electric-dipole spin resonance at acceptor centers. ${ }^{16}$

Electrical detection of a single-electron spin precession is attractive because of high sensitivity of electrical measurements. ${ }^{19}$ Moreover, electrical detection can be performed locally, e.g., by a single-electron $\operatorname{transistor}^{20}$ (SET) or just by measuring the time-dependent potential induced on the gates around the dot holding spin.

To estimate the magnitude of the effect, we consider an electron in a square well defined in a quantum wire. The potential at a distance $r$ from the dot has the order of magnitude $\varphi(r) \approx P_{1} / \epsilon r^{2}$ with $P_{1}$ given by Eq. (9). For an estimate, taking $L=20 \mathrm{~nm}, r=50 \mathrm{~nm}, \epsilon=13$, and $L / \xi \approx 1$, we obtain

$$
\varphi(r) \approx\left(\mu B / \Delta_{1}\right) \times 0.1 \mathrm{mV} .
$$

The detection of a signal with the amplitude (17) oscillating at the Zeeman frequency is certainly feasible. ${ }^{19}$

We expect a stronger effect in the two other situations considered above. In a parabolic $\operatorname{dot}^{21}$ the dipole is typically larger than in a square well, mainly because of its smooth dependence on level number (Fig. 2). In the case of nonKramers level crossing, the effect is enhanced due to the absence of the small factor $\mu B / \Delta_{n}$. The leading effect is dipolar for asymmetric and quadrupolar for symmetric dots. Although exact estimates are problematic because of a large number of independent parameters, we expect the effect in 
this case to be stronger than Eq. (17). The electric signal arising near avoided crossings can also be used to detect level intersections at constant charge in the Coulomb blockade regime.

If spin precession is excited by a resonant external field [electron spin resonance (ESR)], care should be taken to separate the spin-electric signal from the excitation. One possibility is to employ a pulsed ESR excitation and detect precession signal "ringing" after each pulse. Slow spin relaxation times of up to few microseconds reported by Fujisawa et al. $^{22}$ (see also Ref. 2) will simplify the task.

Another possible way is to use thermal excitation of spin precession, i.e., to work at relatively weak magnetic fields $1 / \tau_{2} \ll \mu B \leqq k_{\mathrm{B}} T$, with $\tau_{2}$ the spin dephasing time. Since both spin states, as well as their superpositions, are populated in thermal equilibrium, no external ESR excitation is required in this case. The spin-electric signal will give rise to a narrow band noise forming a peak of width $1 / \tau_{2}$ at the Zeeman frequency. The noise peak value is $\approx \varphi(r) \tau_{2}^{1 / 2}$. With $\tau_{2}=1 \mu \mathrm{s}$ we estimate the peak noise signal as $(\mu B / \Delta)$ $\times 0.1 \mu \mathrm{V} / \mathrm{Hz}^{1 / 2}$.

A narrow-band noise of this form, with a peak at $\omega$ $=\omega_{Z}$, was discovered experimentally by Manassen et al. ${ }^{23}$ in scanning tunneling microscope (STM) current detected near paramagnetic centers on Si surfaces. One can speculate, based on the above, that the SO-induced electric field modulates the tunnel barrier for STM current in vacuum, effectively turning the STM into a spin detector. A recent explanation by Balatsky and Martin, ${ }^{24}$ also based on SO coupling, predicts a current modulation of $\delta I / I \propto I$, while our mechanism remains effective even for $I \rightarrow 0$. Therefore, these mechanisms can be easily distinguished experimentally.

The spin-electric coupling discussed above leads to $a$ new mechanism of spin relaxation in a quantum dot surrounded by metallic electrodes. The low-frequency electric field of the dipole $\mathbf{P}(t)$ penetrates inside the metal, where it can transfer the excitation energy $\hbar \omega_{Z}$ to the Fermi system. This mechanism is dissipationless because quasiparticles acquire energy during passage near the surface and then dissipate it somewhere far away.

The interaction takes place within the screening length $r_{s}$ near the metal surface, where the screened potential is $\widetilde{\varphi}(z, \rho)=-\partial_{z} \varphi(\rho) r_{s} e^{-z / r_{s}}$ [here $\rho$ and $z$ are the coordinates along and perpendicular to the surface, and $\partial_{z} \varphi(\rho)$ is a normal derivative]. The spin relaxation rate can be found from the golden rule:

$$
W=\frac{e^{2}}{\hbar} \iint 2 \operatorname{Im} \mathcal{K}\left(\omega_{Z}, \mathbf{r}_{1}, \mathbf{r}_{2}\right) \widetilde{\varphi}\left(\mathbf{r}_{1}\right) \widetilde{\varphi}\left(\mathbf{r}_{2}\right) d^{3} \mathbf{r}_{1} d^{3} \mathbf{r}_{2},
$$

with $\mathcal{K}\left(\omega_{Z}, \mathbf{r}_{1}, \mathbf{r}_{2}\right)$ the two-point density correlator in the metal and $\mathbf{r} \equiv(z, \rho)$. A finite temperature adds the factor (1 $\left.-e^{-\hbar \omega_{Z} / k_{B} T}\right)^{-1}$. Below we consider $k_{\mathrm{B}} T \ll \hbar \omega_{Z}$.

In the case of specular boundary conditions on a metal surface, using the method of images, the correlator $\mathcal{K}$ near the surface can be related with that in the bulk: $\mathcal{K}_{12}=K_{12}$ $+K_{12^{\prime}}$, where $2^{\prime}$ is a mirror image of the point 2 . We then rewrite Eq. (18) as $W=\left(e^{2} / \hbar\right) \Sigma_{\mathbf{k}}|\widetilde{\varphi}(\mathbf{k})|^{2} \operatorname{Im} K\left(\omega_{Z}, \mathbf{k}\right)$. In a clean metal, using $\operatorname{Im} K(\omega, \mathbf{k})=(\pi / 2) \nu \omega /|\mathbf{k}| v_{F}$, with $\nu$ the density of states,

$$
\begin{aligned}
W & =\frac{e^{2} \nu}{4 \hbar} \omega_{Z} \int\left(\partial_{z} \varphi\right)_{z=0}^{2} d^{2} \rho \int\left(\frac{2 r_{s}^{2}}{1+k^{2} r_{s}^{2}}\right)^{2} \frac{d k}{v_{F}|k|} \\
& \approx\left(e^{2} / h v_{F}\right) \ln \left(r / r_{s}\right)\left(\operatorname{Pr}_{s} / e r^{2}\right)^{2} \omega_{Z} .
\end{aligned}
$$

The logarithm arises due to particles incident at small angles that interact more strongly with the screened dipole field.

For a diffusive metal, with $K(\omega, \mathbf{k})=-\nu D \mathbf{k}^{2} /\left(D \mathbf{k}^{2}\right.$ $-i \omega)$, integrating over the normal component of $\mathbf{k}$, we obtain

$$
\begin{aligned}
W & =\frac{e^{2} \nu r_{s}^{4}}{2 \pi^{2} \hbar D} \int \operatorname{Im} \frac{i \omega_{Z}\left|\partial_{z} \varphi(\mathbf{q})\right|^{2}}{\left(\mathbf{q}^{2}-i \omega_{Z} / D\right)^{1 / 2}} d^{2} \mathbf{q} \\
& \simeq \begin{cases}\left(P r_{s} / r^{2}\right)^{2}\left(\omega_{Z} / D\right)^{1 / 2} / \hbar, & \omega_{Z} \gg D / r^{2}, \\
\left(P r_{s} / r^{2}\right)^{2}\left(\omega_{Z} r / D \hbar\right) \ln \left(D / r^{2} \omega_{Z}\right), & \omega_{Z} \ll D / r^{2}\end{cases}
\end{aligned}
$$

( $\mathbf{q}$ is the parallel-to-surface component of $\mathbf{k}$ ). We find that diffusion enhances $W$ by the number $N$ of returns to the surface during the coherence time, estimated as $(N$ $\simeq \min \left[\left(\omega_{Z} \tau\right)^{-1 / 2}, r / l\right]$, with $\tau$ and $l$ the elastic mean free time and path. These bounds restrict the diffusion time to $\omega_{Z}^{-1}$ and the displacement of diffusing quasiparticles to the distance $r$ from the dot, respectively.

Estimating the relaxation rate (19) with the above parameter values, one has $W \simeq(\mu B / \Delta)^{2} \times 10^{-6} \omega_{Z}$. Recently, electron spin relaxation due to coupling to fluctuating magnetic fields ${ }^{4}$ and to nuclear spins ${ }^{25}$ was considered. Although a direct numerical comparison is difficult due to a wide spread of parameter values, Eq. (21) of Ref. 4 gives a number similar to ours obtained for $\mu B / \Delta \simeq 10^{-2}$. In the case of nonKramers level crossing, due to the absence of the factor $\mu B / \Delta$, the electric mechanism can dominate. Generally, the electrical and magnetic spin relaxation rates depend on different combinations of parameters and, therefore, should be considered as complementary mechanisms. We note also that the estimated spin relaxation time is long compared to $\omega_{Z}^{-1}$, allowing for many cycles of free spin precession.

In summary, the SO-induced electric field around a freely precessing spin can be employed for single-spin detection. It may also significantly contribute to spin relaxation. The reverse spin-electric effect is also of interest in view of spintronics applications. It can serve as a mechanism for independent spin monitoring and control in different dots by local electric field sources.

This work is supported by the MRSEC Program of the National Science Foundation under Grant No. DMR 9808941 (L.L.) and by DARPA/SPINS, Office of Naval Research Grant No. 000140010819 (E.R.). 
*Email: erashba@mailaps.org

${ }^{1}$ E. I. Rashba and V. I. Sheka, in Landau Level Spectroscopy, edited by G. Landwehr and E. I. Rashba (North-Holland, Amsterdam, 1991), p. 131.

${ }^{2}$ S. A. Wolf, D. D. Awschalom, R. A. Buhrman, J. M. Daughton, S. von Molnar, M. L. Roukes, A. Y. Chtchelkanova, and D. M. Treger, Science 294, 1488 (2001); S. Das Sarma, J. Fabian, X. Hu, and I. Žutić, Solid State Commun. 119, 207 (2001).

${ }^{3}$ H.-A. Engel and D. Loss, Phys. Rev. B 65, 195321 (2002).

${ }^{4}$ A. V. Khaetskii and Yu. V. Nazarov, Phys. Rev. B 64, 125316 (2001).

${ }^{5}$ F. G. Pikus and G. E. Pikus, Phys. Rev. B 51, 16928 (1995).

${ }^{6}$ O. Voskoboynikov, C. P. Lee, and O. Tretyak, Phys. Rev. B 63, 165306 (2001); E. N. Bulgakov and A. F. Sadreev, JETP Lett. 73, 505 (2001); M. Governale, Phys. Rev. Lett. 89, 206802 (2002); M. Valín-Rodriguez, A. Puente, L. Serra, and E. Lipparini, Phys. Rev. B 66, 235322 (2002).

${ }^{7}$ V. V. Kveder, V. Ya. Kravchenko, T. R. Mchedlidze, Yu. A. Osip'yan, D. E. Khmel'nitskii, and A. I. Shalynin, JETP Lett. 43, 255 (1986).

${ }^{8}$ A. V. Moroz and C. H. W. Barnes, Phys. Rev. 60, 14272 (1999).

${ }^{9}$ S. Datta and B. Das, Appl. Phys. Lett. 56, 665 (1990).

${ }^{10}$ J. Nitta, T. Akazaki, H. Takayanagi, and T. Enoki, Phys. Rev. Lett. 78, 1335 (1997); G. Engels, J. Lange, T. Schapers, and H. Luth, Phys. Rev. B 55, R1958 (1997).

${ }^{11}$ Y. Sato, T. Kita, S. Gozu, and S. Yamada, J. Appl. Phys. 89, 8017 (2001)

${ }^{12}$ L. J. Cui, Y. P. Zeng, B. Q. Wang, Z. P. Zhu, L. Y. Lin, C. P. Jiang, S. L. Guo, and J. H. Chu, Appl. Phys. Lett. 80, 3132 (2002).

${ }^{13}$ D. Grundler, Phys. Rev. Lett. 84, 6074 (2000).
${ }^{14}$ P. Pfeffer and W. Zawadzki, Phys. Rev. B 59, R5312 (1998); J. A. Majewski, P. Vogl, and P. Lugli, in Proceedings of the 25th International Conference on the Physics Semiconductors, Osaka, 2000, edited by N. Miura and T. Ando (Springer, Berlin, 2001), p. 791.

${ }^{15}$ J. A. Folk, S. R. Patel, K. M. Birnbaum, C. M. Marcus, C. I. Duruoz, and J. S. Harris, Phys. Rev. Lett. 86, 2102 (2002); B. I. Halperin, A. Stern, Y. Oreg, J. N. H. J. Cremers, J. A. Folk, and C. M. Marcus, ibid. 86, 2106 (2001).

${ }^{16}$ E. I. Rashba and V. I. Sheka, Sov. Phys. Solid State 6, 114, 451 (1964).

${ }^{17}$ L. P. Rokhinson, L. J. Guo, S. Y. Chou, and D. C. Tsui, Phys. Rev. Lett. 87, 166802 (2001).

${ }^{18}$ J. A. Folk, C. M. Marcus, R. Berkovits, I. L. Kurland, I. Aleiner, and B. L. Altshuler, Phys. Scr. T90, 26 (2001).

${ }^{19}$ R. C. Ashoori, Nature (London) 379, 6564 (1996); L. Kouwenhoven and C. M. Marcus, Phys. World 11(6), 35 (1998).

${ }^{20}$ R. J. Schoelkopf, P. Wahlgren, A. A. Kozhevnikov, P. Delsing, and D. E. Prober, Science 280, 1238 (1998); M. H. Devoret and R. J. Schoelkopf, Nature (London) 406, 1039 (2000); N. B. Zhitenev, T. A. Fulton, A. Yacoby, H. F. Hess, L. N. Pfeiffer, and K. W. West, ibid. 404, 473 (2000).

${ }^{21}$ A similar enhancement should occur in any shallow dot.

${ }^{22}$ T. Fujisawa, Y. Tokura, and Y. Hirayama, Phys. Rev. B 63, 081304 (2001).

${ }^{23}$ Y. Manassen, R. J. Hamers, J. E. Demuth, and A. J. Castellano, Phys. Rev. Lett. 62, 2531 (1989).

${ }^{24}$ A. V. Balatsky and L. Martin, cond-mat/0112407 (unpublished).

${ }^{25}$ A. V. Khaetskii, D. Loss, and L. I. Glazman, Phys. Rev. Lett. 88, 186802 (2002). 